\title{
Das Südwestdeutsche Brustcentrum (SWBC) Heidelberg - ein Netzwerk zur Qualitätssteigerung in der Diagnostik und Therapie des Mammakarzinoms
}

\author{
Andreas Schneeweiss Jürgen Wacker Christof Sohn \\ Südwestdeutsches Brustcentrum Heidelberg, Germany
}

Trotz steigender Inzidenz sinkt die Brustkrebsmortalität in den westlichen Industrienationen aufgrund eines gesteigerten «Brustbewusstseins» - damit früherer Brustkrebsdiagnose und der Leitlinien-orientierten Therapie. In interdisziplinären Tumorkonferenzen müssen individuell zugeschnittene $\mathrm{Be}$ handlungsstrategien festgelegt werden, deren Komplexität durch die Flut neuer Erkenntnisse aus kontrollierten Studien rasch zunimmt. Um trotzdem in Zeiten knapper werdender Ressourcen die Versorgung von Brustkrebspatientinnen zu optimieren, bedarf es dynamischer Strukturen zur raschen und flächendeckenden Implementierung neuer Standards, für die unabhängige Qualitätskontrolle sowie die nachhaltige und objektive Öffentlichkeitsarbeit. Die ideale Antwort auf diese Anforderungen ist ein flächendeckendes Netzwerk eng kooperierender, regionaler Spezialisten, hier dargestellt am Beispiel des Südwestdeutschen Brustcentrums (SWBC) Heidelberg.

Nach zirka 2-jähriger, intensiver Vorbereitung unter Mithilfe des Westdeutschen Brustcentrums (WBC) in Düsseldorf und Partnern aus der Industrie mit Diskussionsrunden, Seminaren und Hospitationen erfolgte Ende 2003 der vertragliche Zusammenschluss der ersten 6 akademischen Lehrkrankenhäuser bzw. Kreiskrankenhäuser in der Region Rhein-Neckar (KH Bruchsal, KH Salem Heidelberg, KH Schwetzingen, KH Sinsheim, KH St. Josef Heidelberg, KH Weinheim) und der Universitäts-Frauenklinik Heidelberg zum SWBC Heidelberg. Bis Januar 2006 sind weitere 6 Akutkrankenhäuser (KH Buchen, KH Erlenbach, KH Mosbach, Städtisches Klinikum Baden-Baden (3 Kliniken)) und eine Rehaklinik (Sigel-Klinik Bad Schönborn) hinzugekommen. Voraussetzung für die Aufnahme war und ist die an den Leitlinien der Arbeitsgemeinschaft Gynäkologische Onkologie (AGO) der Deutschen Krebsgesellschaft (DKG) orientierte Behandlung von Patientinnen mit Mammakarzinom, die strukturierte und überwachte Datenerfassung im Onkologischen Dokumentationssystem (ODS) easy der Firma Asthenis und die Teilnahme an der unabhängigen Qualitätskontrolle durch externes Benchmarking, welches alle 6 Monate durch das WBC Düsseldorf erfolgt.

Führendes Ziel des SWBC Heidelberg ist die Optimierung von Diagnostik, Therapie und Nachsorge bei Patientinnen mit Mammakarzinom. Dies wird erreicht durch zeitnahe Aktualisierung verbindlicher Arbeitsanweisungen (sogenannte SOPs, standard operating procedures), durch wöchentliche Tumorboards, regelmäßige Fortbildungen, Hospitationen und Seminare sowie das unabhängige Benchmarking der in einem einheitlichen System erfassten und monitorierten Daten. Das SWBC Heidelberg unterstützt seine Mitglieder bei allen Bestrebungen der Qualitätssteigerung insbesondere den Zertifizierungen zum Brustzentrum nach ISO 9001 durch DKG, Deutsche Gesellschaft für Senologie (DGS) und TÜV. Dieser Maßnahmenkatalog führt in der Summe zu Wettbewerbsvorteilen, die sich in einer verstärkten Zuweisung von Patientinnen sowohl mit primärem als auch metastasiertem Mammakarzinom widerspiegeln. Durch die steigenden Patientenzahlen erfolgt eine weitere Effizienz- und Qualitätssteigerung. Im Jahr 2006 werden erstmals über 1000 Patientinnen mit primärem Mammakarzinom innerhalb des SWBC Heidelberg behandelt werden. Diese hohen Patientenzahlen erlauben zudem über garantierte Rekrutierungsfrequenzen die Durchführung und Leitung multizentrischer Studien. Somit besteht die Möglichkeit, schon vor der Zulassung potentiell überlegene Methoden und Therapien anzubieten, die außerhalb des Netzwerkes zum Teil erst Jahre später zur Verfügung stehen. Zur Zeit leitet das SWBC Heidelberg zusammen mit dem Deutschen Krebsforschungszentrum (DKFZ) Heidelberg eine der weltweit innovativsten Multicenterstudien zur primär systemischen Chemotherapie beim Mammakarzinom.

\begin{tabular}{ll}
\hline KARGER & @ 2006 S. Karger GmbH, Freiburg \\
Fax +497614520714 & Accessible online at: \\
$\begin{array}{l}\text { E-mail Information@Karger.de } \\
\text { www.karger.com }\end{array}$ & www.karger.com/brc
\end{tabular}

PD Dr. Andreas Schneeweiss 


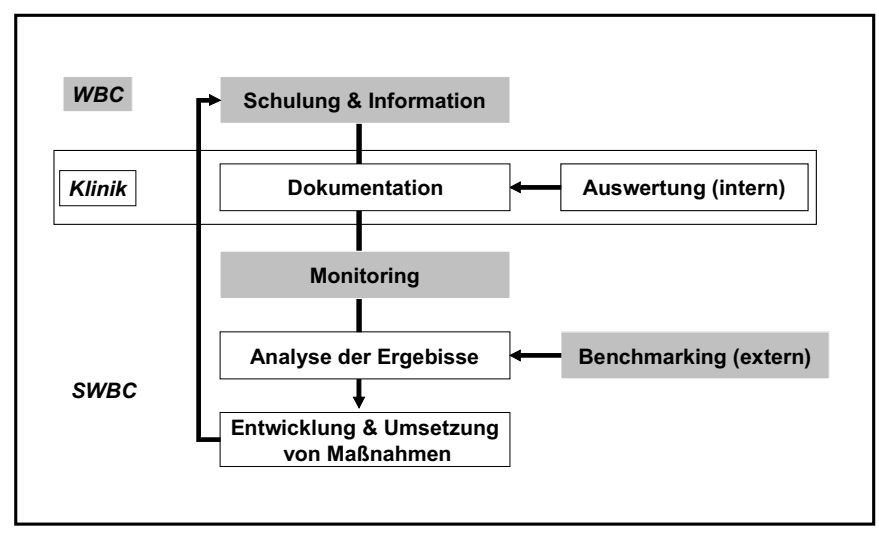

Abb. 1. Qualitätssteigerung durch unabhängiges Benchmarking qualitativ hochwertiger, extern überwachter Daten. WBC: Westdeutsches Brustcentrum Düsseldorf; SWBC: Südwestdeutsches Brustcentrum Heidelberg.

Diese Studie soll abschließend beweisen, dass anhand des Genexpressions-Profils des Primärtumors eine individuell adaptierte Chemotherapie bestimmt werden kann, die einen maximalen Nutzen verspricht. Die Vorhersage (Prädiktion) eines Therapieansprechens wird Unter-, Über- und Fehlbe- handlungen stark reduzieren. Über große, innovative Studien können auch in erheblichem Umfang Drittmittel eingeworben werden, die knappe Personal- und Sachmittelbudgets aufstocken.

Trotz dieser vielfältigen Aufgaben und Möglichkeiten ist die Organisationsstruktur unseres Netzwerkes sehr schlank, dynamisch und damit kostengünstig. Neben einem mit einer Halbtageskraft besetzten Sekretariat trifft sich der wissenschaftliche Beirat, das zentrale Leitungsorgan des SWBC Heidelberg, alle 6-8 Wochen zur Beiratssitzung. In diesem Beirat hat jedes Mitglied eine Stimme. Er ist verantwortlich für die Qualitätssicherung (Festlegung von Standards, Diskussion der Ergebnisse des Benchmarking, Abb. 1), die Organisation regelmäßiger Fortbildungen, die Öffentlichkeitsarbeit (Homepage, Pressearbeit, Organisation von Informationsveranstaltungen), Planung und Durchführung klinischer Studien und translationaler Forschungsprojekte sowie die Fortentwicklung des Netzwerkes (Aufnahme neuer Mitglieder).

Unsere Erfahrungen belegen, dass durch Netzwerke wie das SWBC Heidelberg der Einzelne (sowohl Patient als auch Mitglied oder Mitarbeiter) immer gewinnt ohne seine Eigenständigkeit und Entscheidungsfreiheit einzubüßen. 